\title{
Structure basis for the unique specificity of medaka enteropeptidase light chain
}

\section{Dear Editor,}

Enteropeptidase (enterokinase, EC 3.4.21.9) is a serine protease, which shows a specific cleavage of its substrates at the C-terminal of the recognition site (Asp) ${ }_{4}$ Lys (Zheng et al., 2009). Because of the unique specificity, enteropeptidase could be used as a tool for the production of recombinant fusion proteins. Especially the recombinant enteropeptidase light chain (EPL), which contains a catalytic domain, is of large interest to be applied in biopharmaceutical industry (Lu et al., 1997).

Enteropeptidase has been cloned from several sources, including bovine (Kitamoto et al., 1994), porcine (Matsushima et al., 1994), humans (Kitamoto et al., 1995), mouse (Yuan et al., 1998), and rat (Yahagi et al., 1996). For the high availability, the recombinant bovine enteropeptidase light chain (BEPL) is now most commonly used. However, it is known that BEPL does not exhibit high stringency in its specificity for the canonical target sequence $D_{4} K$ (Liew etal., 2007). For instance, Shahravan et al. showed that BEPL also cleaved at unexpected DR and SR sites in AhR6-C/EBP protein (Shahravan et al., 2008). Recently, the enteropeptidase from medaka had been reported to be a more effective tool, because of its much stricter specific for the $\mathrm{D}_{4} \mathrm{~K}$ sequence compared to its mammalian counterparts (Ogiwara and Takahashi, 2007). Therefore, we sought to solve the crystal structure of the light chain of medaka enteropeptidase (MEPL), and gain insights into the determinants for its stricter specificity.

MEPL shares high sequence similarity with other EPL classes, which have been structurally identified in previous reports (Fig. S1). Therefore, the crystal structure of MEPL was determined by molecular replacement using the bovine enteropeptidase light chain (PDB entry 1EKB, 53.7\% amino acid identity) as the search model and refined to $2.0 \AA$ resolution. The structure of MEPL displays a typical $\alpha / \beta$ trypsin -like serine protease fold (Fig. 1A). It consists of two sixstranded $\beta$-barrels $(\beta 1-\beta 6$ and $\beta 7-\beta 12)$, either of which makes up about one half of the entire molecule. Both $\beta$ barrels are arranged in a Greek-key-pattern containing $\alpha$ helices at the middle of each barrel with a third $\alpha$-helix located at the C-terminus. The surface potentials of MEPL reveal an equal distribution of charged amino acids on protein surface although the region near the active center has a predominantly negative potential (Fig. 1B).
Superimposing of MEPL with the bovine enteropeptidase light chain resulted in an rmsd of $0.79 \AA$ for the $C^{a}$ coordinates (Fig. 1C). The differences are mainly located in the loop regions, such as the so-called '131-loop' that connects strand $\beta 7$ and $\beta 8$. MEPL also contains, relative to bovine enzyme, an additional small $3_{10}$-helix between the strand $\beta 4$ and $\beta 5$. Furthermore, the catalytic triad of the unliganded medaka enteropeptidase superimposes well with the bovine enzyme in complex with a trypsinogen-activation peptide analogue (Lu et al., 1999). However, in secondary structure, the active centers do not show any obvious differences between the two enzymes, which intimates that the stricter specificity of MEPL may depend on the distinct amino acid sequence and unique intramolecular interactions.

The comparison of the catalytic centers between MEPL, BEPL and HEPL (human enteropeptidase light chain, PDB entry $4 D G J$, amino acid identity $55 \%$ ), provide an understanding of the mechanism of substrate specificity functioned by these different enzymes (Fig. 2A and 2B).

In the structure of BEPL with a trypsinogen-activation peptide $\left(V{ }_{4} K\right)$, the side chain of Lys-P1 inserts deeply into a specific pocket, at the bottom of which Asp181 neutralizes the terminal amino group (amino acid residues of peptidyl substrates customarily are numbered P1, P2, P3, etc. (Schechter and Berger, 1967)). The structure of this cleft determinates that the $\mathrm{P} 1$ site of substrates could only be lysine or arginine (Lu et al., 1999). This specific pocket consists of three parts: the strand $\beta 11$, the so called "174loop" that connects strand $\beta 9$ and $\beta 10$, and the "208-loop" that connects strand $\beta 11$ and $\beta 12$. The main differences locate at the N-terminus of "208-loop", while the sequences of strand $\beta 11$ and "174-loop" are highly conservative. Compared with BEPL and HEPL, MEPL shows much less activity for the peptide substrates with arginine at P1 site, which indicates its restriction for the entrance of arginine residues. And according to the structural details, we supposed that the variations of MEPLH24, MEPLE136, MEPLV209, MEPLG210 and MEPLR213 may play curial role in the tension changes of the loop chains around the pocket of catalytic center.

In MEPL structure, MEPLE136 makes three hydrogen bonds with MEPLR213, while there are no similar interactions in BEPL because it is BEPLL213, BEPLY136 in these positions as well as the same situation for the HEPL 


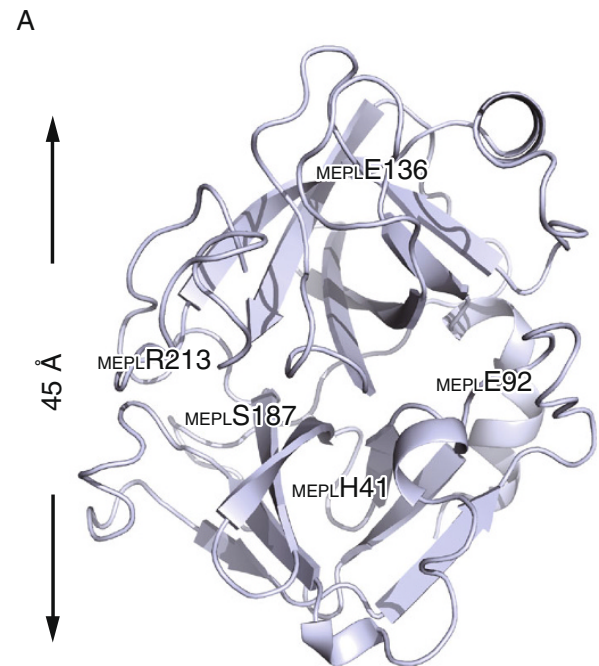

B

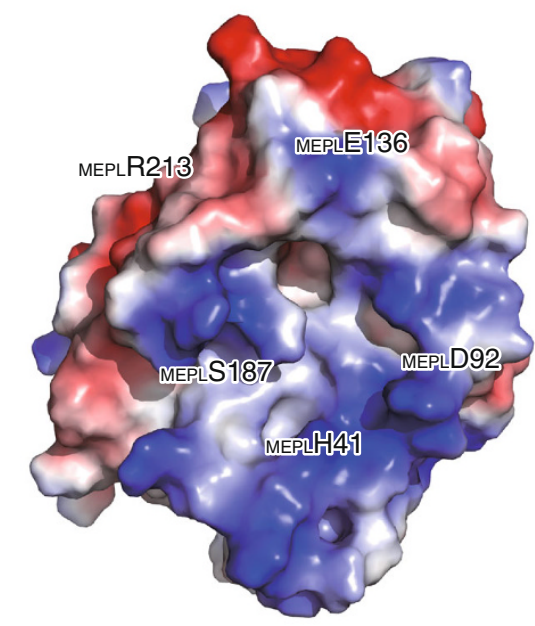

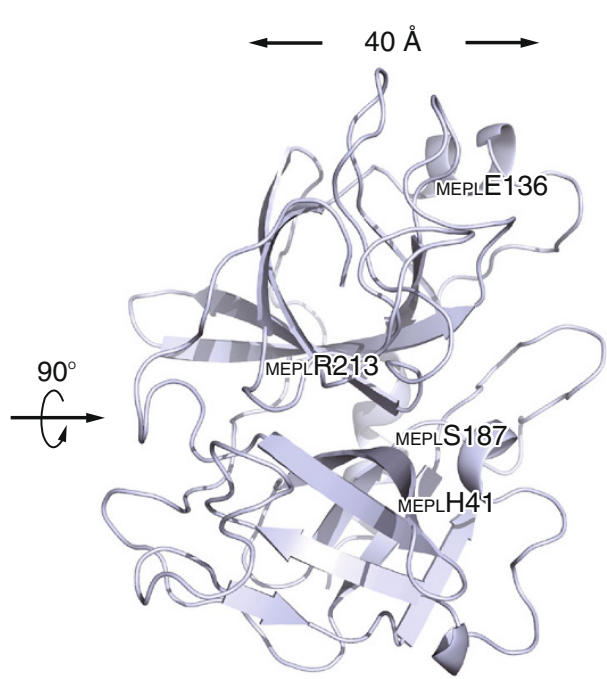

C

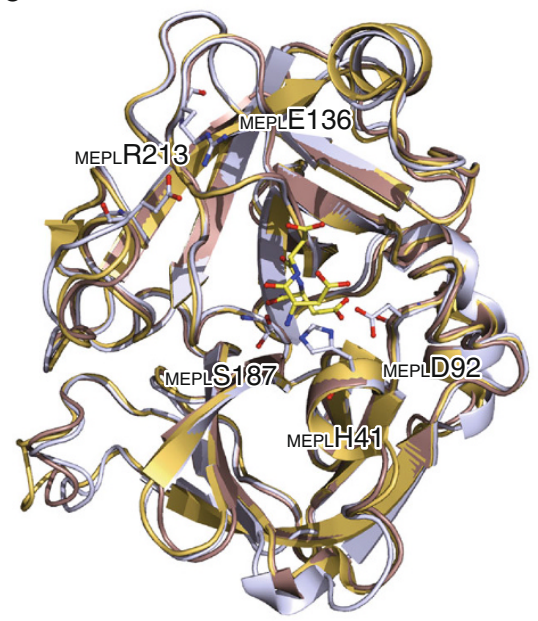

Figure 1. Structure of MEPL. (A) Overall structure of MEPL. The structure of MEPL is shown as ribbon diagrams in two orientations: Front view, looking into the catalytic center; side view, besides the catalytic center. The MEPL molecules are colored as blue-white and several key residues have been labeled. (B) Surface representation and vacuum electrostatic potential of MEPL (where red $=$ electronegative, white = neutral, blue = electropositive). (C) Structural variations of enteropeptidases. A single enteropeptidase molecular of medaka, bovine, and human are shown. The medaka enteropeptidase molecule is colored blue-white, the bovine enteropeptidase is colored salmon, and the human enteropeptidase is colored yellow, respectively. Structural variations are shown with Red Box. Residues in the catalytic center are show in stick. The residues have been renumbered according to the sequence of MEPL.

structure (Fig. 2A). Although the interactions are a little far distance from the catalytic center $(8 \AA)$, these hydrogen bonds may help anchoring the "208-loop", restricting the electron density size of substrate. In addition, MEPLV209 and MEPL $G 210$ with short side chains are covered and fixed by the side chains of four residues (MEPLE136, MEPLE163, MEPLR213 and MEPLR216), reducing the mobility of "208loop" and strand $\beta 11$ in MEPL. However, the amino acid residues at the same positions in BEPL and HEPL are exposed on the surface of the proteins, and therefore possess higher flexibility (Figs. 2A and S2). Moreover, we observed that the imidazole group of ${ }_{\text {MEPL }} \mathrm{H} 24$ could form a hydrogen bond with the main chain oxygen atom of MEPL $G 185$, which could help improving the structural rigidity of "174-loop" (Fig. 2B). Nevertheless, there are no such interactions in BEPL and HEPL because it is BEPLQ24 and HEPLL24 at this position, respectively. Over all, the unique residue interactions in MEPL may reduce the flexibility of the specific pocket, restricting the entrance of arginine residue which has larger side chain.

In order to study the impacts of these residues on MEKLsubstrate interaction, 3 residues in MEPL were replaced by those in BEPL respectively (H24Q, E136Y and R213L), and a combined mutant E136Y/R213L was built. Then, the 

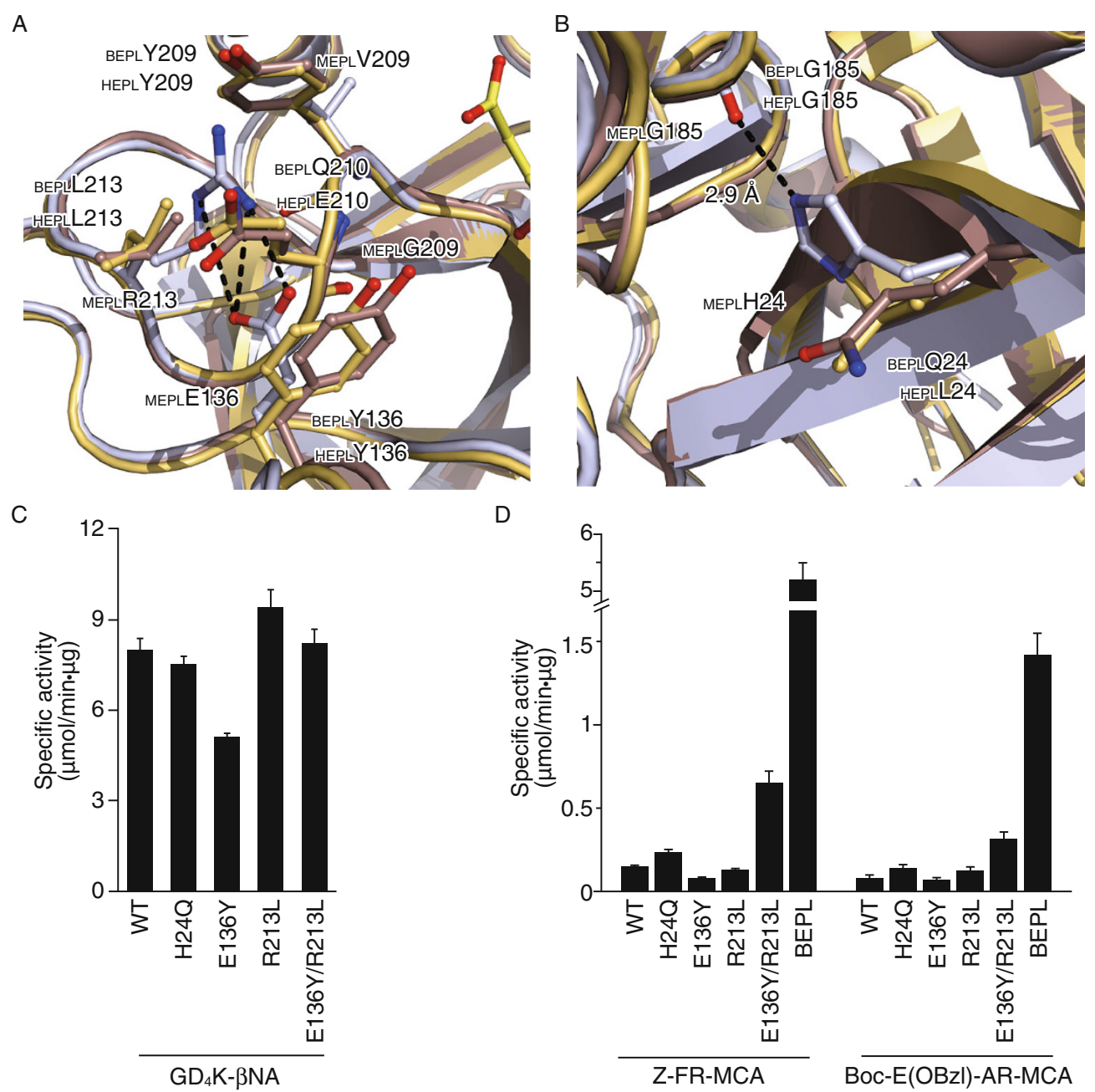

Figure 2. Comparison of the catalytic center in MEPL, BEPL and HEPL, and specific assay of MEPL variants. (A and B) View of detailed two regions considered as playing crucial role in the specificity mechanism. Key residues are shown as colored sticks (blue-white MEPL, salmon BEPL and yellow HEPL, respectively). (C) Recombinant MEPL variants were assayed by using GD4K$\beta N A$ as specific substrate. (D) Recombinant MEPL variants were assayed by using Z-FR-MCA and Boc-E(OBzI)-AR-MCA as unspecific substrates.

specific activities of the four variants were assayed by using the specific substrate $\mathrm{GD}_{4} \mathrm{~K}-\beta N A$ and the non-specific substrates Boc-E(OBZI)-AR-MCA and Z-FR-MCA. The results showed that three mutants (H24Q, R213L and E136Y/ R213L) exhibited significantly increased activity for the MCAcontaining substrates, while retaining $\mathrm{GD}_{4} \mathrm{~K}-\beta \mathrm{NA}$ hydrolyzing activity (Fig. $2 \mathrm{C}$ and $2 \mathrm{D}$ ). This result indicated the importance of the two interactions (H24-G185 and E136R213) for the specificity of enteropeptidase. Nevertheless, these mutations did not raise the activities of MEPL on unspecific substrates to the level of BEPL, which meant there should be other facts affecting the substrate-selectivity of MEPL. The low enzyme activity of mutant E136Y for both GD4K- $\beta N A$ and the MCA-containing substrates meant that the amino acids in position 136 might affect the activity of enteropeptidase. And it was also been provided by the kinetic studies (Table S3).

Taken together, our data on cleavage of peptide substrates, faithfully supported by crystal structures, present an extraordinary example of fine adjustment of enzyme mechanism. Of note, this finding can be directly applied in the reengineering of other enteropeptidases, for instance BEPL and HEPL, to improve the specificity.

\section{FOOTNOTES}

This work was supported by the National Basic Research Program (973 Program) (Nos. 2010CB833605 and 2012CB724502).

We thank the staff members at the Photon Factory, Japan Synchrotron Radiation Facility for their generous help on X-ray data collection. 
Jin Xu, ShiHu, Xiaoze Wang, Ziye Zhao, Xinyue Zhang, Hao Wang, Dapeng Zhang, and Yajun Guo declare that they have no conflict of interest.

Jin $\mathrm{Xu}^{1,3}$, Shi $\mathrm{Hu}^{2,3}$, Xiaoze Wang ${ }^{4}$, Ziye Zhao ${ }^{3,5}$, Xinyue Zhang ${ }^{3,6}$, Hao Wang ${ }^{2,3,5}$, Dapeng Zhang ${ }^{2,3,5 \bowtie}$, Yajun Guo ${ }^{2,3,5 \bowtie}$

${ }^{1}$ School of Pharmacy, Shanghai Jiao Tong University, Shanghai 200240, China

2 International Joint Cancer Institute, Second Military Medical University, Shanghai 200433, China

${ }^{3}$ State Key Laboratory of Antibody Medicine and Targeting Therapy and Shanghai Key Laboratory of Cell Engineering and Antibody, Shanghai 201203, China

${ }^{4}$ PLA General Hospital Cancer Center, PLA Postgraduate School of Medicine, Beijing 100853, China

${ }^{5}$ College of Pharmacy, Liaocheng University, Liaocheng 252000, China

${ }^{6}$ Medical Biotechnology Institute, Soochow University, Suzhou 215007, China

$\bowtie$ Correspondence: zhangdapeng@cmablab.org (D. Zhang), yjguo@smmu.edu.cn (Y. Guo)

\section{OPEN ACCESS}

This article is distributed under the terms of the Creative Commons Attribution License which permits any use, distribution, and reproduction in any medium, provided the original author(s) and the source are credited.

\section{REFERENCES}

Kitamoto Y, Veile RA, Donis-Keller H, Sadler JE (1995) Biochemistry 34:4562-4568

Kitamoto Y, Yuan X, Wu Q, McCourt DW, Sadler JE (1994) Proc Natl Acad Sci USA 91:7588-7592

Liew OW, Jenny Chong PC, Lim YZ, Ang CX, Amy Lau YC et al (2007) Biochimie 89:21-29

Lu D, Yuan X, Zheng X, Sadler JE (1997) J Biol Chem 272:3129331300

Lu D, Futterer K, Korolev S, Zheng X, Tan K et al (1999) J Mol Biol 292:361-373

Matsushima M, Ichinose M, Yahagi N, Kakei N, Tsukada S et al (1994) J Biol Chem 269:19976-19982

Ogiwara K, Takahashi T (2007) Proc Natl Acad Sci USA 104:70217026

Schechter I, Berger A (1967) Biochem Biophys Res Commun 27:157-162

Shahravan SH, Qu X, Chan IS, Shin JA (2008) Protein Expr Purif 59:314-319

Yahagi N, Ichinose $M$, Matsushima $M$, Matsubara $Y$, Miki $K$ et al (1996) Biochem Biophys Res Commun 219:806-812

Yuan X, Zheng X, Lu D, Rubin DC, Pung CY et al (1998) Am J Physiol 274:G342-G349

Zheng XL, Kitamoto Y, Sadler JE (2009) Front Biosci (Elite Ed) $1: 242-249$

Electronic supplementary material The online version of this article (doi:10.1007/s13238-013-0008-x) contains supplementary material, which is available to authorized users.

Jin $\mathrm{Xu}$ and Shi Hu contribute equally to this work. 